AIAA J., vol. 34, no. 11, 1996, pp. 2432-2434

\section{Simulation of Three-Dimensional Symmetric and Asymmetric Instabilities in Attachment-Line Boundary Layers}

\author{
Ronald D. Joslin ${ }^{\dagger}$ \\ NASA Langley Research Center \\ Hampton, Virginia 23681-0001
}

Introduction

ON a swept wing, contamination along the leading edge, Tollmien-Schlichting waves, stationary or traveling crossflow vortices, and/or Taylor-Görtler vortices can cause the catastrophic breakdown of laminar to turbulent flow, which leads to increased skin-friction drag for the aircraft. The discussion in this Note will be limited to disturbances which evolve along the attachment line (leading edge of swept wing). If the Reynolds number of the attachment-line boundary layer is greater than some critical value, then the complete wing is inevitably engulfed in turbulent flow. Essentially, there are two critical Reynolds number points that must be considered. The first is for small-amplitude disturbances, and the second is for bypass transition.

Summarized in Table 1, the experimental and theoretical results agree for the critical Reynolds number where small-amplitude disturbances become unstable on the attachment line. ${ }^{1-4}$ Accounting for all linear terms, and using an eigenvalue problem approach, Hall et al. ${ }^{5}$ studied the linear stability of disturbances in the attachment-line boundary-layer flow called swept Hiemenz flow, which is sketched in Fig. 1. By assuming instability modes which were periodic along the attachment line, the calculations by Hall et al. ${ }^{5}$ agreed with the experiments and with the direct numerical simulations of Spalart ${ }^{6}$, Theofilis $^{7}$, Jiménez et al. ${ }^{8}$, and Joslin. ${ }^{9,10}$

For large-amplitude disturbances, turbulence decays below some critical Reynolds number and transition to turbulence will occur above this point. At this critical point, termed bypass Reynolds number, transition bypasses the conventional linear instability breakdown process. Summarized in Table 2, the experiments show that disturbances are damped for $R_{\theta}<100$ and the flow becomes turbulent for $R_{\theta}>100$. (Refs. 1 and 11-14).

Hall and Malik ${ }^{15}$ attempted to explain this discrepancy between linear theory and the turbulent suppression limits by studying the nonlinear disturbances using weakly nonlinear theory and temporal DNS. Subcritical instability was observed in the computations; how-

$\dagger$ Leader, Laminar Flow Control Project Team, Fluid Mechanics and Acoustics Division. Member AIAA. ever, this subcritical growth did not provide the connection between linear instability and the contamination regions.

Note, the wide gap between the linear critical Reynolds number of $R_{\theta} \simeq 245$ and the turbulent suppression critical Reynolds number of $R_{\theta} \simeq 100$. Bridging this gap is important for wing design. The present study will use direct numerical simulations to validate a linear 2D-eigenvalue prediction method based on parabolized stability equations by Lin and Malik ${ }^{16}$. This method is considered because it suggests that a number of symmetric and asymmetric modes exist and are stable or unstable on the attachment line depending on the Reynolds number. If validated, the approach would predict a number of modes which are linearly damped in the Reynolds number regime 100 to 245; however, these modes may grow nonlinearly and provide an explanation to this region.

Table 1. Critical Reynolds numbers for attachment-line instabilities.

\begin{tabular}{|c|c|}
\hline Experiment & Critical $R_{\theta}$ \\
\hline Cumpsty \& Head $^{1}$ & 245 \\
Pfenninger \& Bacon $^{2}$ & 240 \\
Poll $^{3,4}$ & 230 \\
\hline Calculations $^{2}$ & 245 \\
\hline
\end{tabular}

Table 2. Experimental critical points for attachment-line turbulence suppression.

\begin{tabular}{|c|c|}
\hline Experiment & Bypass $R_{\theta}$ \\
\hline Pfenninger $^{11}$ & 100 \\
Gregory \& Love $^{12}$ & $95-98$ \\
Gaster $^{13}$ & $88-104$ \\
Cumpsty \& Head $^{1}$ & 100 \\
Poll $^{14}$ & 100 \\
\hline
\end{tabular}

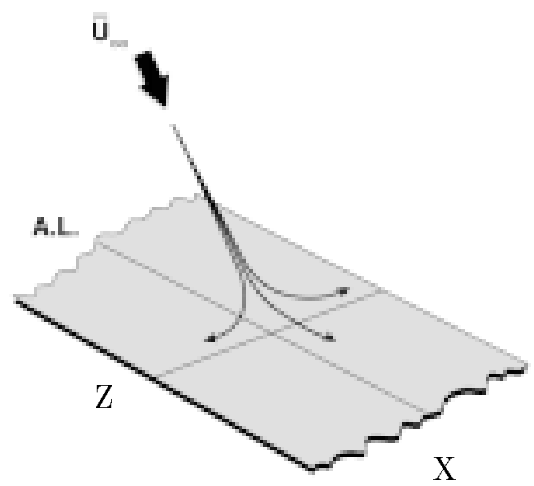

Fig. 1. Sketch of attachment-line region of swept Hiemenz flow. 


\section{Problem Formulation}

In general, the velocities $\underline{\tilde{u}}=(\tilde{u}, \tilde{v}, \tilde{w})$ and the pressure $\tilde{p}$ are solutions of the incompressible, unsteady Navier-Stokes equations. The instantaneous velocities $\underline{\tilde{u}}$ and the pressure $\tilde{p}$ may be decomposed into base-flow velocities $\underline{U}=(U, V, W)$ and pressure $P$ and disturbance velocities $\underline{u}=(u, v, w)$ and pressure $p$. The base flow, originally described by Hall et al. ${ }^{5}$, is referred to as a swept Hiemenz flow. The disturbance is found by solving the 3D incompressible Navier-Stokes equations (in disturbance form)

$$
\begin{gathered}
\frac{\partial \underline{u}}{\partial t}+(\underline{u} \cdot \nabla) \underline{u}+(\underline{U} \cdot \nabla) \underline{u}+(\underline{u} \cdot \nabla) \underline{U}=-\nabla p+\frac{1}{R} \nabla^{2} \underline{u} \\
\nabla \cdot \underline{u}=0
\end{gathered}
$$

with boundary conditions

$$
\underline{u}=0 \text { at } y=0 \text { and } \underline{u} \rightarrow 0 \text { as } y \rightarrow \infty
$$

A Cartesian coordinate system $\underline{x}=(x, y, z)$ is used in which $x$ is aligned with the attachment line, $y$ is wall normal, and $z$ corresponds to the direction of flow acceleration away from the attachment line. A boundary-layer thickness is defined in the $y z$-plane as $\delta=\sqrt{\nu L / W_{o}}$; a Reynolds number, as $R=U_{o} \delta / \nu ; U_{o}, V_{o}, W_{o}$ are velocity scales, and $L$ is the length scale in the flow-acceleration direction $z$. If the attachment line is assumed to be infinitely long, the velocities become functions of $z$ and $y$ only, and the similarity solution can be found.

\section{Numerical Method of Solution}

The 3D DNS code as described by Joslin ${ }^{10}$ is used for the present validation study. High-order finite- and central-differences are used in the attachment-line $(x)$ direction, and Chebyshev series are used in the wallnormal $(y)$ and flow-acceleration $(z)$ directions. For time marching, a time-splitting procedure was used with implicit Crank-Nicolson differencing for normal diffusion terms; an explicit three-stage Runge-Kutta method was used for the remaining terms.

The disturbances are assumed to be from the discrete spectrum, which exponentially decay with distance from the wall. At the wall, in the far field, and at the flow-acceleration boundaries, homogeneous Dirichlet conditions are imposed. The base flow is used for the inflow boundary condition.

\section{Symmetric and Asymmetric Instability Approach}

Recently, Lin and Malik ${ }^{16,17}$ has shown with theory that both symmetric and asymmetric instabilities are present in incompressible and compressible swept Hiemenz flow. The solutions posed by Lin and Malik ${ }^{16}$ took the form

$$
\{u, v, w\}(x, y, z, t)=\{u, v, w\}(y, z) e^{i(\alpha x-\omega t)}
$$

Substituting this form into the Navier-Stokes equations leads to a system of partial differential equations in the flow-acceleration and wall-normal directions. The boundary conditions for the $z$ boundaries took the form: Symmetric:

$$
\begin{gathered}
\frac{\partial u}{\partial z}=\frac{\partial v}{\partial z}=w=0 \quad \text { at } \quad z=0 \\
\{u, v\}(y, z)=\{u, v\}(y,-z), \quad w(y, z)=-w(y,-z) \\
\text { at } z=z_{\text {max }}
\end{gathered}
$$

Asymmetric:

$$
\begin{gathered}
u=v=\frac{\partial w}{\partial z}=0 \quad \text { at } \quad z=0 \\
\{u, v\}(y, z)=-\{u, v\}(y,-z), \quad w(y, z)=w(y,-z) \\
\text { at } z=z_{\text {max }}
\end{gathered}
$$

For the simulations, the entire attachment-line region is included within the computational domain, and therefore, the boundary conditions at $z=0$ are not needed.

The theory suggests that the most unstable modes follow the sequence: symmetric (S1), asymmetric (A1), symmetric (S2), etc. where the growth rates of modes are $S 1>A 1>S 2>A 2>S 3 \ldots$, without exception. This theory and modal growth ordering were recently confirmed by Fedorov ${ }^{18}$ using an asymptotic theory. Although according to the Fedorov analysis, the validation of a single mode implies the validation of all modes, here the first two dominant modes are simulated.

From the results of the Lin-Malik technique, the wavenumber and growth rate for the first three modes at $R=700\left(R_{\theta} \simeq 282\right)$ and $\omega=0.1017$ are shown in Table 3 . The simulation of a pure mode will prove difficult because the discrimination of the wavenumbers would be difficult. The theoretical results suggest that the previous simulations of "discrete modes" are in fact spectrally rich. To use suction and blowing to generate the $\mathrm{S} 1$ mode in the absence of the $\mathrm{S} 2$ mode is probably not possible. However, a discriminating factor can be attributed to the phase relation between the symmetric versus asymmetric modes across the attachment line and in the flow-acceleration direction. This difference is obvious from the $z=0$ boundary conditions (4) and (6). Hence, simulations could discriminate between symmetric and asymmetric modes. 
AIAA J., vol. 34, no. 11, 1996, pp. 2432-2434

Table 3. Lin and Malik ${ }^{16}$ eigenvalues for swept Hiemenz flow at $\mathbf{R}=\mathbf{7 0 0}$ and $\omega=0.1017$.

\begin{tabular}{|c|c|c|}
\hline Mode & $\alpha_{r}$ & $\alpha_{i}$ \\
\hline $\mathrm{S} 1$ & 0.27481152 & $-0.226959 \times 10^{-2}$ \\
$\mathrm{~A} 1$ & 0.27515243 & $-0.105988 \times 10^{-2}$ \\
$\mathrm{~S} 2$ & 0.27548905 & $+0.148157 \times 10^{-3}$ \\
\hline
\end{tabular}

\section{Results}

The simulations are performed on a grid of 661 points ( $\simeq 60$ points per wavelength) along the attachment line, 81 points in the wall-normal direction, and 25 points in the flow-acceleration direction. The far-field boundary is located at $40 \delta$ from the wall, the computational length along the attachment line is $216 \delta$, and the flow-acceleration boundaries are located $\pm 100 \delta$. The total Cray C-90 cost for each simulation is $13 \mathrm{hrs}$ for 8 periods in time. Separately, the symmetric (S1) and asymmetric (A1) modes were forced using suction and blowing. The phase of the A1 mode in the flow-acceleration direction was determined using the Lin-Malik technique.

In Figs. 2 and 3, the simulation results are compared with the wave growth rates described by the theory (listed in Table 3 ). The agreement is remarkably good considering the differences between the DNS and assumed solution form (4). For the theory, the A1 mode has a constant wavenumber and growth rate in the flow-acceleration direction, while the simulations has a truly three-dimensional instability, and therefore, spectral differences in the $z$-direction are inevitable in this $3 \mathrm{D}$ flow. To make this comparison, the results for the simulation are averaged over the flow-acceleration stations: $z=0$ and $z= \pm 6$. These stations were selected because, as Joslin ${ }^{10}$ shows, the streamlines very near the attachment-line are essentially aligned with the 2D attachment-line flow. The $z= \pm 6$ stations permit a cancelation of any opposing flow-acceleration effects.

\section{Concluding Remarks}

In this study, results are presented for the spatial direct numerical simulations (DNS) of $3 \mathrm{D}$ symmetric and asymmetric disturbances that propagate along the attachment line of swept Hiemenz flow. The comparison between the DNS results and the calculations of Lin and Malik $^{16,17}$ demonstrate that both symmetric and asymmetric modes are present in the attachment-line flow and that the theory adequately predicts these modes and the relative dominance of each mode.

Although the connection between the linear instability $R_{\theta} \simeq 245$ and the turbulent contamination $R_{\theta} \simeq 100$ regions was not definitively explained, it is clear that a wealth of instabilities can be present within this $3 \mathrm{D}$ flow field and that some combination of these modes interacting in a nonlinear manner will likely resolve this regiomoô study.

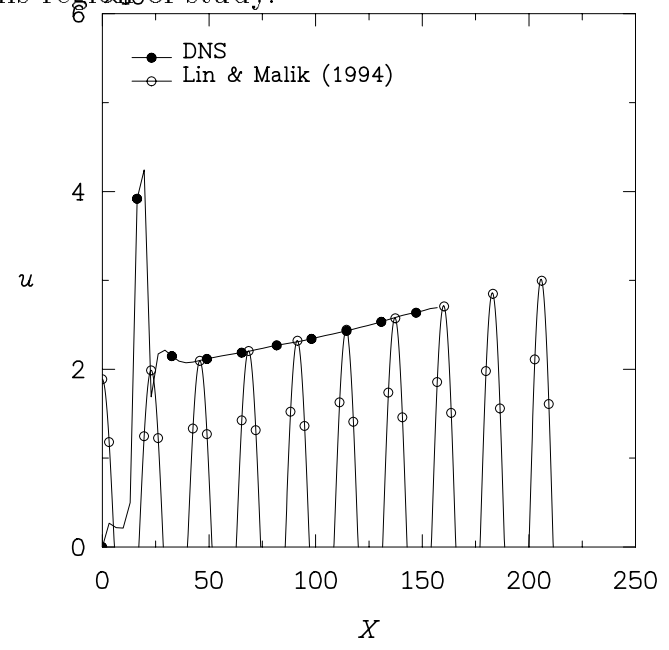

Fig. 2. Symmetric disturbance (S1) growth compared with theory for three-dimensional attachment-line basic flow for $R=700$ and $\omega=$ 0.1017 .

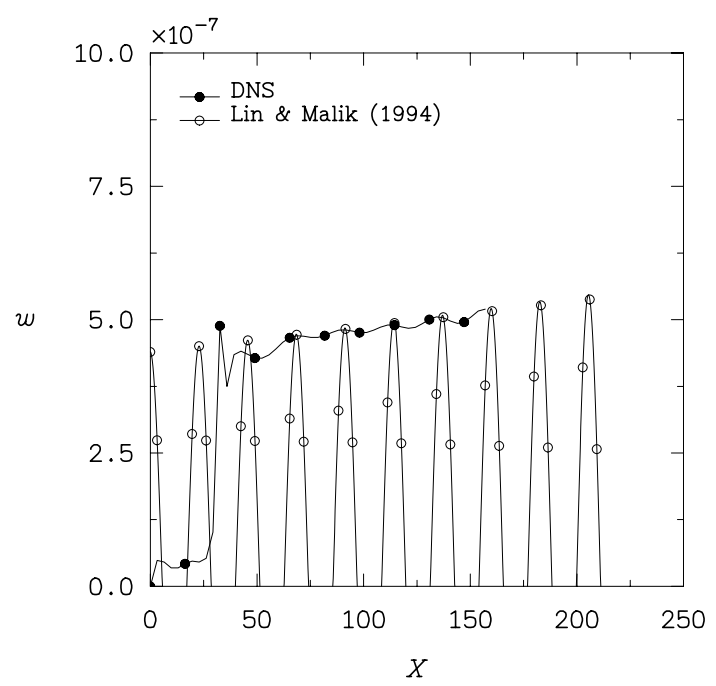

Fig. 3. Asymmetric disturbance (A1) growth compared with theory for three-dimensional attachment-line basic flow for $R=700$ and $\omega=$ 0.1017 .

\section{Acknowledgments}

The author wishes to express his gratitude to Dr. R.-S. Lin, High Technology Corporation, for providing the initial disturbance information for the simulations. 
AIAA J., vol. 34, no. 11, 1996, pp. 2432-2434

\section{References}

${ }^{1}$ Cumpsty, N. A. and Head, M. R. "The calculation of the Three-Dimensional Turbulent Boundary Layer. Part III. Comparison of Attachment-Line Calculations with Experiment," The Aeronautical Quarterly Vol. 20, 1969, pp. 99-113.

${ }^{2}$ Pfenninger, W. and Bacon, J. W., Jr., "Amplified Laminar Boundary-Layer Oscillations and Transition at the Front Attachment Line of a 45 Degree Swept Flat-Nosed Wing with and without BoundaryLayer Suction," Viscous Drag Reduction, (C. S. Wells, ed.), Plenum, 1969, pp. 85-105.

${ }^{3}$ Poll, D. I. A., "Transition in the Infinite Swept Attachment Line Boundary Layer," The Aeronautical Quarterly Vol. 30, 1979, pp. 607-628.

${ }^{4}$ Poll, D. I. A., "Three-Dimensional Boundary Layer Transition via the Mechanisms of AttachmentLine Contamination and Crossflow Stability," LaminarTurbulent Transition, (R. Eppler and H. Fasel, eds.), Springer, Stuttgart, 1980, pp. 253-262.

${ }^{5}$ Hall, P., Malik, M. R. and Poll, D. I. A., "On the Stability of an Infinite Swept Attachment Line Boundary Layer," Proceedings of the Royal Society of London A, Vol. 395, 1984, pp. 229-245.

${ }^{6}$ Spalart, P. R., "Direct Numerical Study of Leading-Edge Contamination," AGARD-CP-438, 1989, pp. 5.1-5.13.

${ }^{7}$ Theofilis, V., "Numerical Experiments on the Stability of Leading Edge Boundary Layer Flow: A TwoDimensional Linear Study," International Journal of Numerical Methods in Fluids, Vol. 16, 1993, pp. 153170 .

${ }^{8}$ Jiménez, J., Martel, C., Agüí, J. C. and Zufiria, J. A., "Direct Numerical Simulation of Transition in the Incompressible Leading Edge Boundary Layer," ETSIA/MF-903, 1990.

${ }^{9}$ Joslin, R. D., "Direct Simulation of Evolution and Control of Nonlinear Instabilities in Attachment-Line Boundary Layers," (AIAA Paper No. 94-0826).

${ }^{10}$ Joslin, R. D., "Direct Simulation of Evolution and Control of Three-Dimensional Instabilities in Attachment-Line Boundary Layers," Journal of Fluid Mechanics Vol. 291, 1995, pp. 369-392.

${ }^{11}$ Pfenninger, W., "Flow Phenomena at the Leading Edge of Swept Wings," Recent Developments in Boundary Layer Research, AGARD 97, May 1965.

${ }^{12}$ Gregory, N. and Love, E. M., "Laminar Flow on a Swept Leading Edge," Final Progress Report, NPL Aero. Memo., Vol. 26, 1965.
${ }^{13}$ Gaster, M., "On the Flow Along Swept Leading Edges," The Aeronautical Quarterly, Vol. 18, 1967, pp. 165-184.

${ }^{14}$ Poll, D. I. A., "Some Observations of the Transition Process on the Windward Face of a Long Yawed Cylinder," Journal of Fluid Mechanics Vol. 150, 1985, pp. 329-356.

${ }^{15}$ Hall, P. and Malik, M. R., "On the Instability of a Three-Dimensional Attachment-Line Boundary Layer: Weakly Nonlinear Theory and a Numerical Simulation," Journal of Fluid Mechanics Vol. 163, 1986, pp. 257-282.

${ }^{16}$ Lin, R.-S. and Malik, M.R., "The Stability of Incompressible Attachment-Line Boundary Layers-A 2DEigenvalue Approach," AIAA Paper No. 94-2372, 1994.

${ }^{17}$ Lin, R.-S. and Malik, M.R., "Stability and Transition in Compressible Attachment-Line Boundary-Layer Flow," SAE Paper No. 952041, 1995.

${ }^{18}$ Fedorov, S., 1995. (personal communication). 